International Journal of Current Microbiology and Applied Sciences

ISSN: 2319-7706 Volume 10 Number 02 (2021)

Journal homepage: http://www.ijcmas.com

Original Research Article

https://doi.org/10.20546/ijcmas.2021.1002.246

\title{
Combining Ability Analysis of Early Maturing Quality Protein Maize (Zea mays L.) Lines and Heterosis of their F1 Hybrids
}

\author{
Preeti Basser*, Shailesh Marker and Kulbhushan Savindra Patil
}

Department of Genetics and Plant Breeding, Sam Higginbottom University of Agriculture Technology and Sciences, Prayagraj, Uttar Pradesh, India

*Corresponding author

\section{Keywords}

Diallel, Heterosis, GCA, SCA, Inbred, Quality Protein Maize

(Zea mays L.)

Article Info

Accepted:

17 January 2021

Available Online:

10 February 2021
The present investigation was carried out with 56 genotypes involving 10 parental inbred lines, their $45 \mathrm{~F}_{1}$ s hybrids and one check (HQPM-5) with an objective to identify the suitable parents and desirable hybrid combinations using diallel mating design (excluding reciprocals) during Rabi 2018-2019 and Kharif 2019 at Field Experimentation Centre, Department of Genetics and Plant Breeding, SHUATS, Prayagraj (Allahabad), U.P. Analysis of variance revealed that the mean sum of squares due to treatments, parents, hybrids and parent vs. hybrids showed significant differences for all traits studied at $1 \%$ level of significance except for Anthesis Silking Interval. The SCA variance were higher than GCA variances for traits viz., anthesis silking interval, plant height and ear height, days to maturity and number of kernel per row indicating predominance of non- additive gene action of these traits. However, the low and moderate values were obtained in the remaining traits. The QPM inbred lines viz., CM-600, HKI-34 (H2), POP31Q-182Q11 and TMT-TROP (QPM) which exhibited good general combiner for at least one trait can be used as donor parents for the accumulation of favourable genes and crosses TARUN-83-1-32 X JP-25W95 and TMT-TROP-(QPM) X CM-138-1 showed highest positive significant SCA effects for grain yield per plant. These hybrids probably have potential as parents of hybrid varieties, as well as for inclusion in breeding programmes. POP-31Q-182Q11 X HKI-193-1, POP-31Q-182Q11 $\mathrm{X}$ TARUN-83-1-32 exhibited positive significant economic heterosis for grain yield and other yield attributing characters over check indicating the possibility of increasing yield by exploiting heterotic potential of these maize genotypes. 


\section{Introduction}

Maize (Zea mays L.) crop has been subjected to pervasive genetic studies than any other crops because of its cross pollinating nature and extremely large genetic variability, which enables it to prosper in tropical, subtropical, and temperate climates. It plays a very important role in human and animal nutrition and its kernel contains $80 \%$ carbohydrates, $10 \%$ protein, $4.5 \%$ oil, $3.5 \%$ fiber and $2 \%$ minerals (Jugenheimer, 1976). It is a good source of calcium and phosphorous. Several hundred million people rely on maize as their principal daily food, for weaning babies, and for feeding livestock. Unfortunately maize (corn) has two significant flaws; it lacks the full range of amino acids, namely lysine and tryptophan, needed to produce proteins, and has its niacin (vitamin $\mathrm{B}_{3}$ ) bound in an indigestible complex. Maize has suboptimal amounts of the essential amino acids tryptophan and lysine, which accounts for its lower status as a protein source (United Nations Food and Agriculture Organization, 1992). The normal maize protein is of poor nutritional quality due to a deficiency in two essential amino acids (lysine and tryptophan) and high leucine-isoleucine ratio. However, the breakthrough in maize production is obtained after the discovery of opaque- 2 mutant maize which alters the amino acid composition and enhances the grain tryptophan and lysine content in maize (Mertz et al., 1964). It promotes evolution of new Quality Protein Maize (QPM) composites such as Ratna, Shakti and Protina. QPM produces $70-100 \%$ more of lysine and tryptophan than the most modern varieties of tropical maize. These two amino acids allow the body to manufacture complete proteins, thereby eliminating wet-malnutrition. In addition tryptophan can be converted in the body to Niacin, which theoretically reduces the incidence of Pellagra. Therefore, it is desirable to replace the present maize hybrids with QPM hybrids which are similar in cultivation, grain yield, potentiality, and tolerance to biotic and abiotic stresses with that of normal maize hybrids.

Development of hybrid variety is the effort that could be considered to increase QPM yield per unit area. Therefore, heterosis and combining ability are the useful parameter for breeding Sugiharto et al., (2018). The magnitude of heterosis provides a basis for genetic diversity and acts as a guide in choosing desirable lines and cross combinations. Heterotic responses can be obtained in the positive and negative directions (+ve and $-v e$ ). Heterosis in negative direction was considered for days to $50 \%$ tasseling, days to $50 \%$ silking, anthesis-silking interval, days to maturity, cob height and plant height and other traits in positive direction of heterosis is desirable. Combining ability of an inbred line depend on its ability to produce superior hybrids in combination with other inbreds and it is one of the powerful tools in identifying the good inbred lines as best combiners that may be used in breeding program either to exploit heterosis or to accumulate productive genes. This approach has been used in identifying suitable maize genotypes for improvement of grain yield and other agronomic traits (Castilo and Goodman, 1989 and Fasahat et al., 2016). It also helps to understand the genetic architecture of various characters that enable the breeder to design effective breeding plan for the future improvement of the existing materials. Therefore, the present study was conducted to estimate the magnitude of heterosis, the GCA effect of parents, SCA effect of hybrids and nature of gene action to explore suitable heterotic hybrid combinations in QPM.

\section{Materials and Methods}

The present investigation was undertaken with ten parental lines viz., HKI-193-1 ( $\left.\mathrm{P}_{1}\right)$, JP-25- 
W95 ( $\left.\mathrm{P}_{2}\right)$, CM-600 ( $\left.\mathrm{P}_{3}\right)$, CM-138-1 ( $\left.\mathrm{P}_{4}\right)$, DMR-N $_{4}\left(\mathrm{P}_{5}\right)$, TARUN-83-1-32 ( $\left.\mathrm{P}_{6}\right)$, LM-13 $\left(\mathrm{P}_{7}\right)$, HKI-34 (H2) ( $\left.\mathrm{P}_{8}\right)$, POP-31Q-182Q11 $\left(\mathrm{P}_{9}\right)$, TMT-TROP-(QPM) $\left(\mathrm{P}_{10}\right)$ which were crossed in diallel mating design (excluding reciprocals) to develop $45 \mathrm{~F}_{1}$ 's hybrids at Field Experimentation Centre, Department of Genetics and Plant Breeding, SHUATS, Prayagraj, Uttar Pradesh during Rabi 20182019 and Kharif 2019 under Randomized Block Design (RBD) with three replications and the experimental field was divided into 3 blocks of equal size with plot with of $3 \mathrm{~m}$ and Size of each bund $0.5 \mathrm{~m}$, the row to row distance and Plant to plant distance $60 \mathrm{~cm}$ and $20 \mathrm{~cm}$ respectively and each line possesses single genotype.

Observations for all traits were recorded on five randomly selected competitive plants from each plot in each replication for all the characters viz. Days to first tassel emergence (50\%), Days to first silk emergence (50\%), Anthesis-silking interval (Days), Plant height (cm), Cob height $(\mathrm{cm})$, Days to maturity, Cob length $(\mathrm{cm})$, Cob girth $(\mathrm{cm})$, Number of kernel rows per cob, Number of kernel per row, 100 kernel weight (g),Grain yield per plant (g/plant) except for days to $50 \%$ silk emergence, days to $50 \%$ tassel emergence and days to maturity where the observation will be recorded on plot basis.

The data recorded during the present investigation was subjected to following statistical analysis i.e. Analysis of variance (Fisher, 1963), Diallel analysis, Average heterosis (Turner, 1953), Heterobeltiosis (Foneska and Patterson, 1968) and Economic heterosis (Meredith and Bridges, 1972).

\section{Results and Discussion}

The present investigation revealed that the mean sum of squares due to treatments, parents, hybrids and parent $v s$. hybrids showed significant differences for all traits studied at $1 \%$ level of significant except for anthesissilking interval, which revealed that there is a wide range of variability among the genotypes suggesting that the genotypes were genetically variant for each other and provide the scope for breeding. Similar findings for analysis of variance for most of the characters in maize were also reported by Sofi and Rather (2006) (Darshan and Marker, 2019). Variances due to General Combining Ability (GCA) and Specific Combining Ability (SCA) were significant for all the characters except Anthesis Silking Interval. This study also revealed the significant differences of general combining ability (GCA) effects of parents and that of specific combining ability (SCA) effects of hybrids. Significant variance due to both GCA and SCA for all important yield attributing characters implied that both additive and non-additive genetic variances are important in controlling the expression of these characters. However, higher magnitude of SCA effects than GCA effects (GCA/SCA<1) were observed for anthesis silking interval, plant height and ear height, days to maturity and number of kernel per row. The relatively smaller proportion of GCA to SCA ratio indicated the predominance of non-additive genetic effects with respect to most of the traits in their inheritance, confirming the results of Aminu and Izge (2013), Patel et al., (2016), Ram et al., (2017) and Darshan and Marker (2019).

\section{GCA effects}

Results concluded (Table 1) that among 10 QPM inbred lines tested for their combining abilities pertaining to different characters under study, line CM-600 recorded significant gca effects in desirable direction for ten different characters viz., days to $50 \%$ tasseling, days to $50 \%$ silking, days to maturity, plant height, cob girth, cob length, kernel row per cob and number of kernel per 
row, test weight, and grain yield per plant followed by HKI-34 (H2) for six characters viz., cob girth., cob length, number of kernel per row, number of kernel row per cob, 100 grain weight and grain yield per plant followed by POP-31Q-182Q11 for plant height, cob girth, number of kernel per row, number of kernel row per cob and grain yield per plant followed by TMT-TROP (QPM) for days to $50 \%$ tasseling, days to $50 \%$ silking, number of kernel per row and CM-138-1 for days to $50 \%$ tasseling, days to $50 \%$ silking and days to maturity.

Thus, the Quality Protein Maize inbred lines viz., CM-600, HKI-34 (H2), POP-31Q182Q11 and TMT-TROP (QPM) which exhibited good general combiner for at least one trait can be used as donor parents for the accumulation of favourable genes (Scaria et al., 2020).

Therefore these lines can be utilized in improvement of the respective traits in any breeding programme wherever hybridization is involved. Due to their good combining ability these lines can be utilized straightaway as parents for production of good hybrids by crossing with other divergent lines and can also be employed in the development of synthetic varieties. These findings are in conformity with the findings of Mostafavi et al., (2012), Aminu et al., (2014) and Scaria et al., (2020).

\section{SCA effects}

The estimates of SCA effects (Table 2) indicated that twelve cross combinations showed negative significant SCA effects for days to $50 \%$ tasseling.

The highest SCA effects for days to $50 \%$ tasseling exhibited by crosses viz., TMTTROP-(QPM) X HKI-193-1 (-4.78**), LM-13 X JP-25-W95 (-4.75**), HKI-34 (H2) X JP-
25-W95 (-4.33**), POP-31Q-182Q11 X HKI$34(\mathrm{H} 2)(-4.19 * *)$ and POP-31Q-182Q11 X TARUN-83-1-32 (-4.00**).

The perusal of SCA effects indicated that eighteen cross combinations showed negative significant SCA effects for days to $50 \%$ silking and the highest SCA effects exhibited by crosses viz., POP-31Q-182Q11 X HKI-34 (H2) (-5.41**), POP-31Q-182Q11 X LM-13 (5.05**), LM-13 X HKI-193-1 (-4.21**) and HKI-34 (H2) X HKI-193-1 (-3.91**). The perusal of SCA effects indicated that eleven cross combinations showed negative significant SCA effects for days to maturity.

The highest SCA effects for days to maturity exhibited by crosses viz., HKI-34 (H2) X HKI-193-1 (-4.13**), POP-31Q-182Q11 X HKI-34 (H2) (-4.08**), POP-31Q-182Q11 X LM-13 (-4.02**) and LM-13 X HKI-193-1 ($\left.3.75^{* *}\right)$ indicating earliness. The highest SCA effects exhibited by crosses TARUN-83-1-32 X JP-25-W95 (62.21**) followed by TMTTROP-(QPM) X CM-138-1 (55.51**), POP31Q-182Q11 X CM-600 (49.09**), POP-31Q182Q11 X HKI-34 (H2) (48.46**) and HKI34 (H2) X CM-600 (30.52**).

These hybrids probably have potential as parents of hybrid varieties, as well as for inclusion in breeding programmes, since they may contribute superior alleles in new populations for high grain yield.

These results are in line with earlier independent studies of Kumar et al., (1998) and Joshi et al., (1998) who reported that maize grain yield and other traits were under the control of non-additive (SCA effects) type of gene action.

Similar results are also found by Junaidul et al., (2015), Patel et al., (2015), Matin et al., (2016), Tulu et al., (2016), Darshan and Marker (2019) and Solomon et al., (2020). 
Table.1 General combining Ability effects of parents for different parameters in Quality Protein Maize

\begin{tabular}{|c|c|c|c|c|c|c|c|c|c|c|c|c|c|c|}
\hline S.No. & Notation & Genotype name & $\begin{array}{c}\text { Days to } \\
50 \% \\
\text { tasseling }\end{array}$ & $\begin{array}{c}\text { Days to } \\
50 \% \\
\text { silking }\end{array}$ & $\begin{array}{l}\text { Anthesis- } \\
\text { silking } \\
\text { interval }\end{array}$ & $\begin{array}{c}\text { Plant } \\
\text { height } \\
(\mathrm{cm})\end{array}$ & $\begin{array}{c}\text { Cob } \\
\text { height } \\
(\mathrm{cm})\end{array}$ & $\begin{array}{l}\text { Days to } \\
\text { maturity }\end{array}$ & $\begin{array}{l}\text { Cob } \\
\text { girth } \\
(\mathrm{cm})\end{array}$ & $\begin{array}{c}\text { Cob } \\
\text { length } \\
(\mathrm{cm})\end{array}$ & $\begin{array}{c}\text { Number } \\
\text { of } \\
\text { kernel } \\
\text { per row }\end{array}$ & $\begin{array}{c}\text { Number } \\
\text { of } \\
\text { kernel } \\
\text { row per } \\
\text { cob }\end{array}$ & $\begin{array}{c}100 \text { seed } \\
\text { weight } \\
\text { (g) }\end{array}$ & $\begin{array}{c}\text { Grain } \\
\text { yield } \\
\text { per } \\
\text { plant } \\
\text { (g/plant) }\end{array}$ \\
\hline 1 & $\mathrm{P}_{1}$ & HKI-193-1 & $1.10 * *$ & $1.21 * *$ & -0.02 & $12.05 * *$ & $-13.57 * *$ & -0.18 & 0.06 & -0.05 & -0.40 & 0.01 & $-1.49 * *$ & $-8.19 * *$ \\
\hline 2 & $\mathrm{P}_{2}$ & JP-25-W95 & -0.03 & 0.10 & 0.14 & $11.08 * *$ & $-4.13 * *$ & -0.15 & $-0.16 *$ & -0.22 & -0.01 & -0.06 & $1.51 * *$ & $5.46 * *$ \\
\hline 3 & $\mathrm{P}_{3}$ & CM-600 & $-0.95 * *$ & $-1.09 * *$ & -0.27 & $23.42 * *$ & $13.32 * *$ & $-1.29 * *$ & $0.20 * *$ & $0.56^{*}$ & $2.42 * *$ & $0.16^{*}$ & $0.75 * *$ & $10.80 * *$ \\
\hline 4 & $\mathrm{P}_{4}$ & CM-138-1 & $-0.53 * *$ & $-0.45^{* *}$ & $0.36^{*}$ & $6.19 * *$ & 0.09 & $-1.35 * *$ & -0.08 & $-0.49^{*}$ & -0.20 & 0.06 & $-0.95 * *$ & $-3.35 * *$ \\
\hline 6 & $\mathrm{P}_{6}$ & TARUN-83-1-32 & 0.272 & $0.32 *$ & -0.10 & $-22.06 * *$ & $1.78 *$ & $1.28 * *$ & $-0.52 * *$ & 0.15 & $-0.61 *$ & $-0.28 * *$ & $0.62 *$ & $-4.34 * *$ \\
\hline 7 & $\mathrm{P}_{7}$ & LM-13 & 0.21 & 0.21 & -0.21 & $10.90 * *$ & $-4.46 * *$ & 0.28 & $-0.15^{*}$ & -0.20 & $-1.47 * *$ & $-0.21 *$ & -0.19 & $-5.81 * *$ \\
\hline 8 & $\mathrm{P}_{8}$ & HKI-34 (H2) & 0.21 & 0.18 & 0.06 & $5.14 * *$ & $7.91 * *$ & $0.78 * *$ & $0.38 * *$ & $0.51 *$ & $1.23 * *$ & $0.57 * *$ & $0.52 *$ & $8.88 * *$ \\
\hline 9 & $\mathrm{P}_{9}$ & POP-31Q-182Q11 & 0.18 & -0.17 & -0.10 & $-3.01 *$ & -0.59 & 0.20 & $0.32 * *$ & 0.26 & $0.91 * *$ & $0.21 * *$ & -0.19 & $2.60 *$ \\
\hline 10 & $\mathrm{P}_{10}$ & TMT-TROP-(QPM) & $-0.47 * *$ & $-0.40 * *$ & 0.17 & $3.01 *$ & -0.66 & -0.10 & $0.19 *$ & 0.11 & $0.77 * *$ & 0.07 & -0.31 & -0.11 \\
\hline
\end{tabular}

$* *$ Significant at $1 \%$ and *Significant at $5 \%$ level 
Table.2 Specific combining Ability effects of crosses for different parameters in Quality Protein Maize

\begin{tabular}{|c|c|c|c|c|c|c|c|c|c|c|c|c|c|}
\hline $\begin{array}{l}\text { S.N } \\
\text { o. }\end{array}$ & Genotypes & $\begin{array}{c}\text { Days to } \\
\mathbf{5 0 \%} \\
\text { tasseling }\end{array}$ & $\begin{array}{c}\text { Days to } \\
\mathbf{5 0 \%} \\
\text { silking }\end{array}$ & $\begin{array}{c}\text { Anthesis } \\
\text {-silking } \\
\text { interval }\end{array}$ & $\begin{array}{c}\text { Plant } \\
\text { height } \\
(\mathbf{c m})\end{array}$ & $\begin{array}{c}\text { Cob } \\
\text { height } \\
(\mathrm{cm})\end{array}$ & $\begin{array}{c}\text { Days to } \\
\text { maturity }\end{array}$ & $\begin{array}{l}\text { Cob } \\
\text { girth } \\
(\mathrm{cm})\end{array}$ & $\begin{array}{c}\text { Cob } \\
\text { length } \\
(\mathrm{cm})\end{array}$ & $\begin{array}{l}\text { No. of } \\
\text { kernel / } \\
\text { row }\end{array}$ & $\begin{array}{c}\text { No. of } \\
\text { kernel } \\
\text { row / cob }\end{array}$ & $\begin{array}{c}100 \text { seed } \\
\text { weight } \\
\text { (g) }\end{array}$ & $\begin{array}{c}\text { Grain yield / } \\
\text { plant } \\
\text { (g/plant) }\end{array}$ \\
\hline 1 & JP-25-W95 X HKI-193-1 & 0.19 & 0.51 & -0.45 & $-25.61 * *$ & -10.19 & $2.69 * *$ & $-0.64 *$ & -0.21 & 1.68 & $0.81 * *$ & $-2.30 * *$ & -2.40 \\
\hline 2 & CM-600 X HKI-193-1 & -0.83 & $-1.52 * *$ & 0.10 & -2.43 & 4.69 & 0.77 & -0.19 & -0.12 & $3.09 * *$ & $-1.28 * *$ & $-1.96^{*}$ & $-7.65^{*}$ \\
\hline 3 & CM-600 X JP-25-W95 & -0.16 & -0.41 & 0.71 & 7.05 & -2.31 & $1.47 *$ & 0.04 & -0.60 & -1.64 & -0.09 & -2.55 & $-11.85 * *$ \\
\hline 4 & CM-138-1 X HKI-193-1 & -0.83 & $-1.55^{* *}$ & -0.01 & -0.52 & -0.67 & $1.61 *$ & 0.12 & $3.49^{* *}$ & $2.67 * *$ & 0.30 & -0.15 & 5.65 \\
\hline 5 & CM-138-1 X JP-25-W95 & $-1.86 * *$ & 0.22 & 0.93 & $-28.10 * *$ & $-15.67 * *$ & -0.02 & 0.15 & $-1.99 *$ & $-3.26^{* *}$ & $0.82 * *$ & $-2.64 * *$ & $-13.70 * *$ \\
\hline 6 & CM-138-1 X CM-600 & -0.50 & $-1.46^{* *}$ & -0.51 & 2.34 & -0.83 & 0.38 & 0.37 & -0.58 & $-2.18^{*}$ & $0.73 * *$ & 0.86 & -1.12 \\
\hline 7 & DMR-N ${ }_{4}$ X HKI-193-1 & -0.83 & 1.00 & -0.12 & -3.56 & 2.36 & -0.38 & $-1.74 * *$ & 0.05 & $7.28^{* *}$ & $0.90^{* *}$ & -0.09 & $22.32 * *$ \\
\hline 8 & DMR-N $_{4}$ X JP-25-W95 & $1.44 * *$ & -0.21 & 0.49 & $-20.13 * *$ & $-16.24 * *$ & -0.02 & $1.61 * *$ & -0.11 & $-2.79 * *$ & 0.23 & $2.64^{* *}$ & 0.59 \\
\hline 9 & DMR-N ${ }_{4}$ X CM-600 & -0.22 & $-1.91 * *$ & -0.62 & 0.50 & $-16.41 * *$ & -0.61 & 0.06 & 0.16 & -0.24 & $0.94 * *$ & $-1.75^{*}$ & $-4.05^{*}$ \\
\hline 10 & DMR-N ${ }_{4}$ X CM-138-1 & $-1.58 * *$ & -0.93 & -0.06 & 4.48 & -3.23 & 1.22 & 0.52 & $1.80^{*}$ & $2.26^{*}$ & 0.25 & -0.21 & -7.07 \\
\hline 11. & TARUN-83-1-32 X HKI-193-1 & $-1.25 *$ & 0.07 & 0.07 & $10.87^{*}$ & 7.15 & 0.05 & $0.92 * *$ & $-1.93^{*}$ & $-4.45^{* *}$ & $-0.62 *$ & $8.35^{* * *}$ & 3.69 \\
\hline 12. & TARUN-83-1-32 X JP-25-W95 & 0.38 & 0.35 & 0.35 & $19.42 * *$ & $18.42 * *$ & $-1.58 *$ & $-2.47 * *$ & $2.39 * *$ & $8.13^{* *}$ & $1.33^{* *}$ & $7.56^{* *}$ & $62.21^{* *}$ \\
\hline 13. & TARUN-83-1-32 X CM-600 & -1.28 & 0.90 & 0.90 & 1.80 & -1.08 & -1.16 & $0.73^{* *}$ & 0.92 & -0.78 & $-0.72 *$ & $2.06^{*}$ & -6.11 \\
\hline 14. & TARUN-83-1-32 X CM-138-1 & 0.02 & -0.87 & -0.87 & $-19.95 * *$ & -3.57 & 1.00 & $0.73 * *$ & $1.80^{*}$ & -0.47 & 0.32 & $-2.90^{*}$ & $-4.47 * *$ \\
\hline 15. & TARUN-83-1-32 X DMR-N & -0.30 & 0.01 & 0.01 & 5.27 & $10.04 * *$ & 0.33 & $-0.63^{*}$ & -1.05 & $-3.66 * *$ & -0.26 & -0.42 & $-13.17 * *$ \\
\hline 16. & LM-13 X HKI-193-1 & -0.36 & $-4.21 * *$ & $1.07 *$ & $12.91 * *$ & 21.45 & $-3.75 * *$ & -0.49 & 1.29 & $3.73 * *$ & -0.18 & $3.31^{* *}$ & 22.99 \\
\hline 17. & LM-13 X JP-25-W95 & $-4.73 * *$ & 0.22 & -0.98 & $12.40 * *$ & $8.64 * *$ & $-2.72 * *$ & 0.10 & $-1.95^{*}$ & $-2.74 * *$ & -0.19 & $2.67 * *$ & -4.50 \\
\hline 18. & LM-13 X CM-600 & 0.91 & $1.53 * *$ & 0.90 & $9.44 *$ & 5.20 & -0.63 & $-1.20 * *$ & 0.27 & $3.40^{* *}$ & $0.65^{*}$ & 1.32 & $17.40^{* *}$ \\
\hline 19. & LM-13 X CM-138-1 & 0.55 & $2.17 * *$ & $-1.20 *$ & $9.15^{*}$ & $7.38^{*}$ & -0.13 & $0.84 * *$ & -0.68 & -1.61 & $0.76 * *$ & 0.18 & -3.51 \\
\hline 20. & LM-13 X DMR-N & $1.88^{* *}$ & 0.72 & 0.35 & $40.38 * *$ & 4.94 & 0.19 & -0.25 & 1.43 & $4.78^{* *}$ & $0.97^{* *}$ & -1.51 & 6.19 \\
\hline 21. & LM-13 X TARUN-83-1-32 & 0.16 & -0.71 & 0.21 & 0.08 & $13.20 * *$ & 2.30 & $0.93^{* *}$ & 0.38 & -0.35 & 1.04 & 0.68 & 3.30 \\
\hline 22. & HKI-34 (H2) X HKI-193-1 & -0.55 & $-3.91 * *$ & 0.37 & $26.82 * *$ & 5.22 & $-4.13 * *$ & 0.01 & 0.09 & -0.82 & $1.26 * *$ & $3.11^{* *}$ & $16.43^{* *}$ \\
\hline 23. & HKI-34 (H2) X JP-25-W95 & $-4.33 * *$ & -0.13 & -0.01 & $37.64 * *$ & $15.62 * *$ & -1.11 & 0.72 & -0.62 & -0.76 & -0.07 & $2.78^{* *}$ & 5.42 \\
\hline 24. & HKI-34 (H2) X CM-600 & -0.66 & -0.49 & 0.21 & $29.95 * *$ & $15.18 * *$ & -0.02 & $0.86^{* *}$ & $1.93^{*}$ & $2.51^{* *}$ & 0.49 & $4.83 * *$ & $30.52 * *$ \\
\hline 25. & HKI-34 (H2) X CM-138-1 & -0.36 & -0.52 & $1.10 *$ & $43.06 * *$ & $26.35^{* *}$ & -0.86 & $-2.33 * *$ & 0.53 & 0.69 & $-0.58 *$ & $-3.54 * *$ & $-18.25 * *$ \\
\hline 26. & HKI-34 (H2) X DMR-N & -0.36 & $2.69 * *$ & -0.67 & $54.82 * *$ & $37.58 * *$ & 1.80 & 0.07 & 1.31 & $6.89^{* *}$ & -0.24 & 1.38 & $19.27 * *$ \\
\hline 27. & HKI-34 (H2) X TARUN-83-1-32 & $2.24 * *$ & $3.92 * *$ & 0.18 & $14.32 * *$ & $12.44 * *$ & 1.25 & $-0.69^{*}$ & $1.88^{*}$ & 0.02 & 0.08 & $-2.65^{* *}$ & $-11.57^{* *}$ \\
\hline 28. & HKI-34 (H2) X LM-13 & $1.52 * *$ & $-3.85 * *$ & $1.18 *$ & $16.56 * *$ & $5.80 *$ & $-2.55^{* *}$ & $0.73^{* *}$ & 1.07 & $1.88^{*}$ & 0.26 & -1.22 & -3.99 \\
\hline 29. & POP-31Q-182Q11 X HKI-193-1 & $-3.61 * *$ & $3.89 * *$ & -0.70 & $21.49 * *$ & $-15.45^{* *}$ & 2.38 & 0.35 & -0.38 & $-4.18 * *$ & -0.11 & -0.10 & $-12.15^{* *}$ \\
\hline 30. & POP-31Q-182Q11 X JP-25-W95 & $3.41 * *$ & -0.99 & 0.57 & -6.88 & $-12.05 * *$ & 0.75 & 0.52 & -0.61 & $-2.39 *$ & $1.21 * *$ & 0.34 & -3.07 \\
\hline 31. & POP-31Q-182Q11 X CM-600 & -0.91 & $1.64 * *$ & 0.46 & 5.96 & -0.63 & 0.83 & 0.25 & $2.69^{* *}$ & $5.35^{* *}$ & $0.85 * *$ & $6.54^{* *}$ & $49.09 * *$ \\
\hline 32. & POP-31Q-182Q11 X CM-138-1 & 0.05 & $-1.05^{*}$ & -0.31 & $35.87 * *$ & $12.08 * *$ & 0.66 & 0.38 & 0.65 & $3.59^{* *}$ & -0.36 & $2.41^{* *}$ & $15.97 * *$ \\
\hline
\end{tabular}


Int.J.Curr.Microbiol.App.Sci (2021) 10(02): 2065-2075

\begin{tabular}{|c|c|c|c|c|c|c|c|c|c|c|c|c|c|}
\hline 33. & POP-31Q-182Q11 X DMR-N & -0.61 & $-3.82 * *$ & $1.24 *$ & $39.76^{* *}$ & $26.90 * *$ & $-2.33 * *$ & $-1.73 * *$ & -0.84 & $2.00^{*}$ & -0.15 & 0.43 & 0.63 \\
\hline 34. & POP-31Q-182Q11 X TARUN-83-1-32 & $-4.00 * *$ & $-1.27 *$ & -0.23 & $23.40 * *$ & 2.89 & -0.22 & $-0.82 * *$ & -0.60 & -1.93 & 0.17 & $3.21^{* *}$ & -2.17 \\
\hline 35. & POP-31Q-182Q11 X LM-13 & -0.72 & $-5.05 * *$ & 0.10 & $18.03 * *$ & $10.72 * *$ & $-4.02 * *$ & -0.08 & $2.49 * *$ & -1.07 & $1.15^{* *}$ & -0.93 & $-3.14 * *$ \\
\hline 36. & POP-31Q-182Q11 X HKI-34 (H2) & $-4.19 * *$ & $-5.41 * *$ & -0.92 & -1.91 & $-7.83 * *$ & $-4.08 * *$ & 0.83 & $1.93 *$ & $4.63^{* *}$ & $1.53^{* *}$ & $5.37 * *$ & $48.46^{* *}$ \\
\hline 37. & TMT-TROP-(QPM) X HKI-193-1 & $-4.78 * *$ & 0.44 & -0.20 & $9.42 *$ & 4.45 & -1.25 & 0.13 & 1.31 & -1.32 & $-0.85^{* *}$ & $-3.66 * *$ & $-16.88 * *$ \\
\hline 38. & TMT-TROP-(QPM) X JP-25-W95 & 0.94 & $-1.43 * *$ & -0.59 & $18.45 * *$ & $7.11 *$ & $-1.55^{*}$ & -0.41 & 0.02 & $-3.33^{* *}$ & $-2.46^{* *}$ & -0.85 & $-19.62 * *$ \\
\hline 39. & TMT-TROP-(QPM) X CM-600 & $-1.41 * *$ & $-1.80 * *$ & -0.70 & $-15.63 * *$ & $10.47 * *$ & $-2.80 * *$ & $1.11^{* *}$ & $-2.34 * *$ & $-4.52 * *$ & 0.11 & 1.43 & -6.00 \\
\hline 40. & TMT-TROP-(QPM) X CM-138-1 & $-1.08 *$ & $-1.82 * *$ & -0.48 & $28.73 * *$ & $10.12 * *$ & 0.36 & $1.09 * *$ & -1.43 & $4.85 * *$ & $1.36 * *$ & $8.86^{* *}$ & $55.51 * *$ \\
\hline 41. & TMT-TROP-(QPM) X DMR-N 4 & -0.47 & -0.60 & -0.92 & $31.03 * *$ & 4.47 & -1.30 & $1.33^{* *}$ & -0.11 & 0.12 & 0.43 & $3.87 * *$ & $17.74 * *$ \\
\hline 42. & $\begin{array}{l}\text { TMT-TROP-(QPM) X TARUN-83-1- } \\
32\end{array}$ & $-1.86 * *$ & $-2.05 * *$ & -0.39 & 6.13 & $-12.26 * *$ & $-1.52 *$ & -0.28 & -0.03 & $1.92 *$ & $1.97 * *$ & $3.30 * *$ & $7.62 *$ \\
\hline 43. & TMT-TROP-(QPM) X LM-13 & -0.33 & -0.49 & -0.06 & $28.23 * *$ & -0.10 & 1.00 & 0.05 & 1.36 & $2.17 *$ & 0.28 & -1.24 & 0.22 \\
\hline 44. & TMT-TROP-(QPM) X HKI-34 (H2) & -0.58 & $-1.18^{*}$ & 0.24 & $-36.65 * *$ & $-21.53 * *$ & $2.27 * *$ & 0.50 & -0.33 & -0.51 & -0.54 & 1.41 & 3.9 \\
\hline 45. & $\begin{array}{l}\text { TMT-TROP-(QPM) X POP-31Q- } \\
\text { 182Q11 }\end{array}$ & $2.497 * *$ & $3.28 * *$ & 0.49 & $14.44 * *$ & $16.25 * *$ & -0.19 & $0.58 *$ & 0.11 & 0.66 & 0.48 & $-2.35 * *$ & -4.84 \\
\hline
\end{tabular}

**Significant at $1 \%$ and *Significant at $5 \%$ level 
Table.3 Economic heterosis (Hc) for Quantitative traits in Quality Protein Maize

\begin{tabular}{|c|c|c|c|c|c|c|c|c|c|c|c|c|c|}
\hline $\begin{array}{c}\text { S.N } \\
\text { o. }\end{array}$ & Genotypes & $\begin{array}{c}\text { Days to } \\
50 \% \\
\text { tasseling }\end{array}$ & $\begin{array}{c}\text { Days to } \\
\mathbf{5 0 \%} \\
\text { silking }\end{array}$ & $\begin{array}{l}\text { Anthesis- } \\
\text { silking } \\
\text { interval }\end{array}$ & $\begin{array}{c}\text { Plant } \\
\text { height } \\
\text { (cm) }\end{array}$ & $\begin{array}{c}\begin{array}{c}\text { Cob } \\
\text { height } \\
(\mathbf{c m})\end{array} \\
\end{array}$ & $\begin{array}{c}\text { Days to } \\
\text { maturity }\end{array}$ & $\begin{array}{l}\text { Cob } \\
\text { girth } \\
(\mathbf{c m})\end{array}$ & $\begin{array}{c}\text { Cob } \\
\text { length } \\
(\mathrm{cm})\end{array}$ & $\begin{array}{c}\text { No. of } \\
\text { kernel / } \\
\text { row }\end{array}$ & $\begin{array}{c}\text { No. of } \\
\text { kernel row } \\
\text { / cob }\end{array}$ & $\begin{array}{c}100 \text { seed } \\
\text { weight } \\
\text { (g) }\end{array}$ & $\begin{array}{c}\text { Grain yield / } \\
\text { plant } \\
\text { (g/plant) }\end{array}$ \\
\hline 1 & JP-25-W95 X HKI-193-1 & $-8.19 * *$ & $-8.84 * *$ & $-41.67 *$ & $-9.49 *$ & $-24.08^{* *}$ & -0.36 & -5.60 & -14.42 & -6.55 & 6.52 & -4.81 & 2.06 \\
\hline 2 & CM-600 X HKI-193-1 & $-7.60 * *$ & $-9.39 * *$ & -16.67 & $10.44^{* *}$ & -4.39 & -0.72 & 1.68 & -14.34 & $-15.94 * *$ & 3.26 & -1.98 & 1.38 \\
\hline 3 & CM-600 X JP-25-W95 & $-9.94 * *$ & $-11.60^{* *}$ & -25.00 & $8.28^{*}$ & 3.91 & -1.79 & -1.41 & -12.21 & -0.87 & $-7.61 *$ & 0.24 & 3.55 \\
\hline 4 & CM-138-1 X HKI-193-1 & $-10.53^{* *}$ & $-10.50 * *$ & $-50.00 *$ & 3.29 & -7.26 & -1.79 & 0.43 & $-17.25^{*}$ & $-25.55^{* *}$ & 6.52 & $14.27 *$ & 4.76 \\
\hline 5 & CM-138-1 X JP-25-W95 & $-8.19 * *$ & $-8.29 * *$ & -16.67 & $-18.61 * *$ & $-35.17 * *$ & $-2.87 *$ & -1.90 & $-28.06 * *$ & $-30.13 * *$ & 4.35 & -5.81 & $-22.88 * *$ \\
\hline 6 & CM-138-1 X CM-600 & $-9.94 * *$ & $-11.60 * *$ & -33.33 & 0.26 & $-17.30 * *$ & -1.43 & -3.21 & 6.73 & $-11.14^{*}$ & -1.09 & 5.43 & 1.51 \\
\hline 7 & DMR-N ${ }_{4}$ X HKI-193-1 & $-9.36^{* *}$ & $-9.39 * *$ & $-41.67 *$ & $-10.93 * *$ & $-17.46^{* *}$ & -0.36 & -2.50 & -4.08 & $-17.03 * *$ & $-10.87 * *$ & 3.66 & -2.61 \\
\hline 8 & DMR-N $_{4}$ X JP-25-W95 & $-9.94 * *$ & $-11.05^{* *}$ & $-50.00 *$ & -4.08 & $-18.42 * *$ & -1.79 & -1.36 & -10.08 & $-16.38 * *$ & -5.43 & -0.24 & 2.62 \\
\hline 9 & DMR-N 4 X CM-600 & $-7.60 * *$ & $-8.84 * *$ & -25.00 & $-20.42 * *$ & $-28.39 * *$ & -1.79 & -5.60 & -13.55 & $-25.76^{* *}$ & $-7.61^{*}$ & $17.21 * *$ & 0.23 \\
\hline 10 & DMR-N ${ }_{4}$ X CM-138-1 & $-5.85^{* *}$ & $-7.18 * *$ & -33.33 & $-7.79 *$ & -6.22 & $-2.51^{*}$ & 5.92 & -13.42 & 6.77 & 6.25 & 3.66 & $28.10^{* *}$ \\
\hline 11. & TARUN-83-1-32 X HKI-193-1 & $-8.19 * *$ & $-9.39 * *$ & -33.33 & -4.58 & 4.15 & -1.08 & $-9.95^{* *}$ & $-25.63 * *$ & $-40.17 * *$ & $-10.87 * *$ & 8.28 & $-32.47 * *$ \\
\hline 12. & TARUN-83-1-32 X JP-25-W95 & $-8.19 * *$ & $-9.39 * *$ & $-58.33 * *$ & $-12.56^{* *}$ & $-19.62 * *$ & -1.43 & $-11.85^{* *}$ & -9.30 & $-32.53 * *$ & -5.43 & -1.46 & $-21.87 * *$ \\
\hline 13. & TARUN-83-1-32 X CM-600 & $-7.60 * *$ & $-8.29 * *$ & -8.33 & $8.89 *$ & -1.83 & $-3.23 * *$ & $-8.59 * *$ & -10.34 & $-24.67 * *$ & $-7.61^{*}$ & $25.57^{* *}$ & -2.73 \\
\hline 14. & TARUN-83-1-32 X CM-138-1 & $-9.94 * *$ & $-11.60^{* *}$ & -25.00 & $14.26^{* *}$ & $11.32^{*}$ & $-4.30 * *$ & 2.07 & -2.39 & 3.49 & 6.25 & $48.25^{* *}$ & $88.22 * *$ \\
\hline 15. & TARUN-83-1-32 X DMR-N & $-8.19 * *$ & $-8.29 * *$ & -25.00 & $12.63^{* *}$ & -2.23 & $-2.87 *$ & 0.98 & $-31.58 * *$ & $-38.21 * *$ & $-10.87 * *$ & $51.48^{* *}$ & -1.53 \\
\hline 16. & LM-13 X HKI-193-1 & $-9.94 * *$ & $-10.50^{* *}$ & -16.67 & $8.51^{*}$ & 5.90 & -1.79 & -0.27 & $-20.42 * *$ & $-27.95 * *$ & 2.17 & $11.97 *$ & -6.85 \\
\hline 17. & LM-13 X JP-25-W95 & $-8.19 * *$ & $-7.73 * *$ & -16.67 & $19.14 * *$ & -2.23 & $-3.23 * *$ & -3.59 & -8.34 & -4.59 & 3.26 & -6.11 & -0.28 \\
\hline 18. & LM-13 X CM-600 & $-5.26 * *$ & $-5.52 * *$ & $-58.33 * *$ & $7.75^{*}$ & -6.78 & $-4.66^{* * *}$ & -6.41 & $-24.46^{* *}$ & $-28.38 * *$ & 2.17 & 4.04 & $-16.67 *$ \\
\hline 19. & LM-13 X CM-138-1 & $-7.60 * *$ & $-6.63^{* *}$ & 0.00 & $17.02^{* *}$ & 5.42 & $-4.66^{* *}$ & 5.54 & -13.55 & -3.06 & $7.61 *$ & $12.84^{*}$ & $35.53^{* *}$ \\
\hline 20. & LM-13 X DMR-N & $-7.02 * *$ & $-9.39 * *$ & $-50.00 *$ & $14.07 * *$ & -0.64 & $-7.53 * *$ & -4.67 & $-29.71 * *$ & $-24.24 * *$ & -2.17 & $15.82 * *$ & -5.77 \\
\hline 21. & LM-13 X TARUN-83-1-32 & $-18.13 * *$ & $-17.13^{* *}$ & 8.33 & $17.59 * *$ & $14.59^{* *}$ & $-8.96 * *$ & 3.21 & -9.56 & -3.49 & -3.26 & $18.29 * *$ & $30.55^{* *}$ \\
\hline 22. & HKI-34 (H2) X HKI-193-1 & $-16.96 * *$ & $-17.68 * *$ & 0.00 & $31.43^{* *}$ & $12.60^{*}$ & $-8.96 * *$ & -0.43 & -7.99 & -4.15 & 1.09 & 1.85 & 6.99 \\
\hline 23. & HKI-34 (H2) X JP-25-W95 & $-7.02 * *$ & $-3.87 * *$ & -33.33 & $26.36^{* *}$ & $20.81 * *$ & $-2.87 *$ & -2.88 & -3.78 & $-18.12 * *$ & -4.35 & 4.23 & -7.92 \\
\hline 24. & HKI-34 (H2) X CM-600 & $-5.26^{* *}$ & $-5.52 * *$ & $-58.33 * *$ & $37.10^{* *}$ & $52.63^{* *}$ & -1.43 & -2.83 & -2.22 & $10.92 *$ & -5.43 & $15.79 * *$ & $39.62 * *$ \\
\hline 25. & HKI-34 (H2) X CM-138-1 & $-9.94 * *$ & $-11.05 * *$ & -16.67 & $36.7^{* *}$ & $31.74 * *$ & $-5.38 * *$ & -1.25 & -9.60 & $-12.23 * *$ & $-7.61 *$ & -5.59 & $-17.53^{*}$ \\
\hline 26. & HKI-34 (H2) X DMR-N & $-9.94 * *$ & $-11.05 * *$ & -33.33 & $38.43^{* *}$ & $33.17 * *$ & $-3.94 * *$ & 6.28 & 4.17 & 2.62 & $7.61^{*}$ & $37.64 * *$ & $75.51 * *$ \\
\hline 27. & HKI-34 (H2) X TARUN-83-1-32 & $-10.53 * *$ & $-11.0 * *$ & $-41.67 *$ & $38.16^{* *}$ & $23.52 * *$ & $-5.73 * *$ & -5.87 & -14.12 & -9.17 & 0.00 & $24.47 * *$ & $29.52 * *$ \\
\hline 28. & HKI-34 (H2) X LM-13 & $-18.13 * *$ & $-17.68 * *$ & -25.00 & $35.25^{* *}$ & $11.00^{*}$ & $-9.32 * *$ & -0.71 & -10.43 & $-9.83^{*}$ & $9.78^{* *}$ & $25.49^{* *}$ & $41.68 * *$ \\
\hline 29. & POP-31Q-182Q11 X HKI-193-1 & $-18.13^{* *}$ & $-19.34 * *$ & $-58.33 * *$ & $23.71^{* *}$ & -8.77 & $-9.32 * *$ & $6.63^{*}$ & -0.61 & 5.46 & $10.87^{* *}$ & $44.88^{* *}$ & $96.80^{* *}$ \\
\hline 30. & POP-31Q-182Q11 X JP-25-W95 & $-16.37 * *$ & $-17.68 * *$ & -16.67 & $25.26^{* *}$ & -2.39 & $-9.32 * *$ & 2.34 & -3.87 & $-21.83 * *$ & 6.52 & 6.78 & 0.41 \\
\hline 31. & POP-31Q-182Q11 X CM-600 & $-9.36 * *$ & $-10.50 * *$ & -33.33 & $24.51^{* *}$ & $-11.48^{*}$ & $-3.23 * *$ & 1.14 & $-25.11 * *$ & $-32.53 * *$ & -5.43 & $35.74 * *$ & -1.96 \\
\hline
\end{tabular}


Int.J.Curr.Microbiol.App.Sci (2021) 10(02): 2065-2075

\begin{tabular}{|c|c|c|c|c|c|c|c|c|c|c|c|c|c|}
\hline 32. & POP-31Q-182Q11 X CM-138-1 & $-14.62 * *$ & $-14.36 * *$ & 0.00 & $21.56^{* *}$ & $18.98^{* *}$ & $-4.66 * *$ & $-13.48 * *$ & $-21.46^{* *}$ & $-13.10 * *$ & -6.52 & $14.87^{*}$ & 4.49 \\
\hline 33. & POP-31Q-182Q11 X DMR-N & $-8.77 * *$ & $-9.94 * *$ & $-41.67 *$ & $25.68 * *$ & -6.22 & $-2.51 *$ & -6.09 & -13.99 & $-10.70^{*}$ & $-7.61^{*}$ & $26.33^{* *}$ & $24.81 * *$ \\
\hline 34. & $\begin{array}{l}\text { POP-31Q-182Q11 X TARUN-83-1- } \\
32\end{array}$ & $-7.60 * *$ & $-5.52 * *$ & -16.67 & $17.81 * *$ & -6.62 & -1.79 & 4.02 & 3.95 & 3.93 & $8.70^{*}$ & $49.37^{* *}$ & $94.89 * *$ \\
\hline 35. & POP-31Q-182Q11 X LM-13 & $-9.36^{* *}$ & $-10.50 * *$ & -16.67 & 5.90 & $-30.46 * *$ & $-2.51 *$ & -3.91 & $-19.20 *$ & $-22.49 * *$ & $8.70^{*}$ & $16.47 * *$ & 9.25 \\
\hline 36. & POP-31Q-182Q11 X HKI-34 (H2) & $-2.92 *$ & $-2.76^{*}$ & $-41.67 *$ & $25.23 * *$ & $-34.61 * *$ & -1.08 & $-11.03 * *$ & $-18.68 *$ & $-28.82 * *$ & -3.26 & $13.79 *$ & -8.04 \\
\hline 37. & TMT-TROP-(QPM) X HKI-193-1 & -1.75 & -1.10 & -16.67 & -3.52 & $-12.12 *$ & $-3.94 * *$ & $-10.11 * *$ & $-16.55^{*}$ & $-16.81 * *$ & 1.09 & -2.55 & -9.17 \\
\hline 38. & TMT-TROP-(QPM) X JP-25-W95 & $-8.77 * *$ & $-10.50 * *$ & -33.33 & $-9.11 *$ & $-36.44 * *$ & $-2.51 *$ & 1.79 & -14.29 & $-12.66 * *$ & -5.43 & 11.78 & 11.59 \\
\hline 39. & TMT-TROP-(QPM) X CM-600 & $-7.60^{* *}$ & $-8.29 * *$ & -25.00 & $17.93 * *$ & $-26.63 * *$ & $-3.94 * *$ & -4.29 & -10.17 & $-12.45 * *$ & 0.00 & -8.98 & -14.68 \\
\hline 40. & TMT-TROP-(QPM) X CM-138-1 & $-9.36^{* *}$ & $-9.94 * *$ & $-41.67 *$ & 1.59 & $-40.91 * *$ & $-4.66 * *$ & $-8.37 *$ & $-20.33 * *$ & $-21.18 * *$ & $9.78 * *$ & -9.56 & -7.62 \\
\hline 41. & TMT-TROP-(QPM) X DMR-N & $-6.43 * *$ & $-7.18 * *$ & $-58.33 * *$ & 3.48 & $-19.14 * *$ & $-3.58 * *$ & -1.09 & -15.60 & $-20.52 * *$ & -1.09 & $16.91 * *$ & 9.54 \\
\hline 42. & $\begin{array}{l}\text { TMT-TROP-(QPM) X TARUN-83- } \\
1-32\end{array}$ & $-7.60 * *$ & $-9.39 * *$ & $-50.00 *$ & $8.51^{*}$ & $-19.86^{* *}$ & $-2.87^{*}$ & $10.16^{* *}$ & $-26.50 * *$ & -7.86 & $7.07 *$ & $42.66^{* *}$ & $62.75^{* *}$ \\
\hline 43. & TMT-TROP-(QPM) X LM-13 & $-8.19 * *$ & $-9.39 * *$ & $-50.00 *$ & $-7.56^{*}$ & -4.63 & $-5.73 * *$ & -2.99 & $-27.76^{* *}$ & $-29.69 * *$ & 3.26 & 10.78 & -5.87 \\
\hline 44. & TMT-TROP-(QPM) X HKI-34 (H2) & $-7.60 * *$ & $-9.39 * *$ & $-50.00 *$ & 7.15 & $-18.82 * *$ & $-5.02 * *$ & 5.87 & -13.94 & $-26.86 * *$ & $-20.65^{* *}$ & -3.53 & $-35.03 * *$ \\
\hline 45. & $\begin{array}{c}\text { TMT-TROP-(QPM) X POP-31Q- } \\
\text { 182Q11 }\end{array}$ & $-5.26 * *$ & $-6.63 * *$ & -33.33 & 5.26 & $-22.09 * *$ & $-5.02 * *$ & 4.51 & -6.52 & $-20.74 * *$ & $-8.70^{*}$ & $-17.43^{* *}$ & $-34.99 * *$ \\
\hline
\end{tabular}

**Significant at $1 \%$ and *Significant at $5 \%$ level 


\section{Heterosis}

Cross POP-31Q-182Q11 X HKI-193-1 exhibited highest negative significant economic heterosis (Table 3) for traits viz., days to $50 \%$ tasseling (18.13\%), days to $50 \%$ silking (19.34\%) and Anthesis Silking Interval (58.33\%). Crosses viz., POP-31Q-182Q11 X JP-25-W95, POP-31Q-182Q11 X HKI-193-1 and HKI-34 (H2) X LM-13 recorded highest negative significant heterosis for days to maturity. These crosses can be exploited further in breeding program for developing early maturing varieties. Genotypes which showed early maturity can be of immense use in future breeding program. These findings are in conformity with the findings of Shah et al., (2016) and Matin et al., (2016). The desirable crosses combination POP-31Q-182Q11 X HKI-193-1 and POP-31Q-182Q11 X TARUN83-1-32 showed high magnitude of heterosis over the check variety HQPM-5 for grain yield along with other yield attributing characters. The study of heterotic response revealed that we can adopt the option of developing single cross QPM hybrids from the materials under study that belong to high Quality Protein Maize Remtluangpuii et al., (2020), Darshan and Marker (2019), Solomon et al.,(2020).

\section{Acknowledgements}

Present investigation was done as a part of M.Sc. thesis. The authors are thankful to the Department of Agriculture, Agriculture Educational Research and Government of Uttar Pradesh for providing financial assistance to this project.

\section{References}

Aminu, D. and Izge, A. U. (2013). Gene action and Heterosis for Yield and Yield Traits in Maize (Zea mays L.), Under Drought Conditions in Northern
Guinea and Sudan Savannas of Borno State. Peak Journal of Agricultural Sciences, 1(1):17 - 23.

Aminu, D., Mohammed, S. G., Kabir, B. G. (2014). Estimates of Combining Ability and Heterosis for Yield and Yield Traits in Maize Population (Zea mays L.), under Drought Conditions in the Northern Guinea and Sudan Savanna Zones of Borno State, Nigeria. International Journal of Agriculture Innovations and Research, 2 (5): 2319-1473.

Darshan, S. S. and Marker, S. (2019).Heterosis and combining ability for grain yield and its component characters in quality protein maize (Zea mays L.) hybrids. Electronic Journal of Plant Breeding, 10(1):111118

Gammingi, S. R., Marker, S., Scaria S. and Ansari, S. M. S. (2020). Quantitative studies on heterosis and inbreeding depression in quality protein maize for terminal heat tolerance. Journal of Pharmacognosy and Phytochemistry, 9 (2): 784-788.

Joshi, V. N., Pandiya, N. K. and Dubey, R. B. (1998). Heterosis and Combining Ability for Quality and Yield in Early Maturing Single Cross Hybrids of Maize. Indian Journal of Genetic and Breeding, 58: 519-524.

Junaidul, H. I., Onovo, J. C., Mijinyawa, A. and Abdul K. B. M. (2015). Combining ability in quality protein maize using diallel crosses among five inbred lines. Journal of Natural Sciences Research, 5 (14) 2224-3186.

Kumar, A., Gangashetti, M. G. and Kumar, N. (1998). Gene Effects in Some Metric Traits of Maize. Annual Agricultural Biology Research, 3:139 - 143.

Matin MQ, Rasul MG, Islam A, Mian MA, Ivy NA, Ahmed U (2016). Combining ability and Heterosis in Maize (Zea 
mays L.). American Journal of Bio Science, 6(4):84-90.

Mostafavi, K., Choukan R., Taeb, M., Heravan, E. M and Bihamta, M.M. (2012). Heterotic Grouping of Iranian Maize Inbred Lines Based on Yield Specific Combining Ability in Diallel Crosses and GGE Biplot. Journal of Research in Agricultural Science, 8(2):113- 125.

Patel. P.C. and Kathiria K. B. (2016). Heterosis and combining ability for yield and quality traits in Quality Protein Maize (Zea mays L.) Electronic Journal of Plant Breeding, 7(4): 960-966.

Ramteke, P., Marker, S and Synrem, G. (2017). Gene Action and Combining ability analysis for Grain yield and Quality parameters in Sub-tropical Maize (Zea mays L.). International journal of plant research, 30: 140-148.
Remtluangpuii, H.P. and Marker, S. (2020). Heterosis in early maturing high yielding quality protein maize (Zea mays) hybrids. Journal of Pharmacognosy and Phytochemistry, 9(2): 1561-1563.

Scaria, S., Marker, S., Gammingi, S. R and Karthika, A. (2020). Genetic evaluation of quality protein maize hybrids for heterosis and combining ability for grain yield and its component characters. Journal of Pharmacognosy and Phytochemistry, 9(2): 1101-1105.

Shah L, Rahman H, Ali A, Shah KA, Si H, Xing WS, Lia WS.(2016) Early generation testing for specific combining ability and heterotic effects in maize variety Sarhad white. ARPN Journal of Agricultural Biological and Science, 11(1):42-48.

\section{How to cite this article:}

Preeti Basser, Shailesh Marker and Kulbhushan Savindra Patil. 2021. Combining Ability Analysis of Early Maturing Quality Protein Maize (Zea mays L.) Lines and Heterosis of their F1 Hybrids. Int.J.Curr.Microbiol.App.Sci. 10(02): 2065-2075. doi: https://doi.org/10.20546/ijcmas.2021.1002.246 